\title{
THE ESTIMATION OF THE BACTERICIDAL POWER OF THE BLOOD
}

\author{
By A. A. MILES and S. S. MISRA \\ From the Department of Pathology, British Postgraduate Medical School \\ WITH A NOTE BY J. O. IRWIN \\ Division of Epidemiology and Vital Statistics, London School of \\ Hygiene and Tropical Medicine
}

THE killing power of the blood is usually estimated from the result of cultivating mixtures of whole or defibrinated blood with graded inocula of bacterial culture. A series of culture dilutions made with a constant dilution factor provides what is in effect a logarithmic scale upon which the bactericidal power may be measured in terms of the number of dilutions sterilized (e.g. Mackie \& Finkelstein, 1931). In many tests, however, the bactericidal power of the blood is of a lower order; the blood sterilizes only the highest dilution of culture that contains viable organisms, or the viable organisms in a given dilution undergo only a reduction in numbers. It would be unjustifiable, from the result of two such tests, to infer anything about differences in the bactericidal power of two bloods without some knowledge of the sampling errors inherent in the technique employed.

The absence of growth from a small inoculum in blood cannot be depended upon as an indication of killing, unless very large numbers of fluid cultures are made from each blood-inoculum mixture (Fisher, 1930, section 17). ${ }^{1}$ Within the limits of a practicable technique, accuracy is approached only when methods involving colony counts are used. The investigations described below were directed particularly towards finding reliable quantitative expressions for the killing power of human blood for Staphylococcus aureus, measured by viable counting methods, but many of the principles involved are applicable to bactericidal tests in general.

\section{The ESTIMATION OF THE NUMBER OF BACTERIA INOCULATED}

Two accurate viable counting methods are available: the plate count and the roll-tube count. Both, depending on the incorporation of the culture in molten agar media, involve a number of laborious manipulations. We have used a similar count of the colonies developing from measured drops of culture dilutions seeded on the surface of well-dried agar plates. A few parallel tests have shown that this "surface-viable" count does not differ significantly from counts of colonies developing in the depth of roll-tubed agar. We have not

\footnotetext{
1 All subsequent references to Fisher indicate various sections of the 1930 publication.
} 
pursued this point, for "viability" is a function of the cultural conditions, and provided that these are optimal and standard for the technique employed, the actual level of viability, within reasonable limits, is immaterial. It remains to be tested whether a series of surface-viable counts gives results as consistent as those of the established plate and roll-tube counts.

Method. Dropping pipettes, giving 50 drops of broth to the c.c., were employed. Tenfold dilutions of culture are made, $0 \cdot 1$ c.c. being added to 0.9 c.c. of diluent broth, and a fresh pipette used for each dilution. (For details of the pipette technique and its reliability see Wilson, 1922, 1935.) Two per cent. agar plates, after the routine $24 \mathrm{hr}$. drying at $37^{\circ} \mathrm{C}$. with the lids closed, are dried for a further $2 \mathrm{hr}$. with the lids raised. Except for counts of delicate bacteria, this period may be extended to $6 \mathrm{hr}$. without materially altering the count result. The plates should be dry enough to absorb a 0.02 c.c. drop in 15-20 min., and for this a standard $2 \mathrm{hr}$. drying is usually sufficient. On such plates, $0.02 \mathrm{c.c}$. of broth, dropped from a height of $2.5 \mathrm{~cm}$., spreads over an area $1.5-2 \cdot 0 \mathrm{~cm}$. in diameter. Six to eight dilutions of culture are made, and at the same time each of six plates receives one drop of each of the dilutions in numbered sectors; after the absorption of the drops the plates are incubated in the usual way. The drop areas from the higher concentrations of culture yield circular patches of confluent growth. Counts are made in drop areas containing the largest number of colonies without signs of confluence or of gross diminution in colony size due to overcrowding. The number of colonies is estimated from the mean of the six counts. With large colonies of organisms like Bacterium coli (Kenny et al. 1937), the maximum number obtainable without confluence in the drop area may be as low as 20 , but with small colonies, like those of Staphylococcus aureus after $18 \mathrm{hr}$. growth, counts up to 100 per drop area may be made.

\section{Tests of a suitable medium}

Ideally, the frequencies of constant-volume samples of a thin bacterial suspension containing $0,1,2,3$, etc., living bacteria should conform to a Poisson series $e^{-M}\left(1+M+M^{2} / 2 !+M^{3} / 3 !+\ldots\right.$ etc. $)$, where $M$ is the average bacterial content per sample. The viable counts from such samples will also conform to the Poisson series if (1) each plate offers the same facilities for the development of each organism; (2) the development of any organism is independent of other organisms present, and if development results only in one visible colony; (3) each plate has an equal chance of receiving any organism, and if the organisms are distributed independently. These conditions were enumerated by Fisher et al. (1922) for counts of mixed bacterial populations. They are easier to fulfil for surface-viable counts of pure cultures. (2) is satisfied if there is no overcrowding in the drop areas, and if, as with the strains of Staph. aureus used, the organisms are dispersed singly in the culture. (3) is realized in the technique of diluting and distributing the drops. There remains condition (1), and it follows that, other conditions being satisfied, an imperfection of nutrient qualities of the medium would be reflected, not only in a reduction of the number of bacteria developing into colonies, but in a lessened conformity of the six count values with the ideal Poisson distribution.

The mean count, $M$, and the standard error, s.e., of a count may be estimated from six parallel platings, $x_{1}, x_{2}, \ldots, x_{6}$, by the expressions $m=S(x) / N$, and $\sigma=\sqrt{ }\left[S(x-m)^{2} /(N-1)\right]$ respectively, where $N$ is the number of plates. 
The s.E. of an ideal Poisson distribution is equal to the square root of the mean, and some idea of the conformity of a particular count to the Poisson series can be gained by comparing the calculated s.E. with the expected s.E., $\sqrt{ } m$. But this comparison does not indicate the degree of conformity, and the goodness of the fit of the six-plate count to a Poisson series may be more accurately measured by the $\chi^{2}$ test. $\chi^{2}$ is calculated, according to the formula for small samples of a Poisson series (Fisher, sect. 16), from the relation $\chi^{2}=S(x-m)^{2} / m$. With only six available values of $x$, the resulting $\chi^{2}$ is not a reliable indication of conformity, but the values of $\chi^{2}$ from a number of such counts should fall within assignable limits if the counts themselves are derived from Poisson variates. Parallel counts were made on nutrient agar plates (N.A.P.) prepared according to Hedley Wright's (1933) method, and on the same medium containing $2 \%$ Fildes' extract. In all, sixteen batches of ox-heart extract were

Table I. The distribution of $\chi^{2}$ from surface-viable counts of Staph. aureus on nutrient and Fildes' agar plates

\begin{tabular}{|c|c|c|c|c|c|c|c|}
\hline \multirow[b]{2}{*}{$\chi^{2}$} & \multirow[b]{2}{*}{ Expected } & \multirow[b]{2}{*}{ F.A.P. } & \multirow[b]{2}{*}{ N.A.P. } & \multicolumn{4}{|c|}{ F.A. } \\
\hline & & & & Expected & Total & $m<20$ & $m>35$ \\
\hline $0.00-0.55$ & $0 \cdot 16$ & - & - & 0.94 & 1 & 一 & 1 \\
\hline $0.56-0.75$ & $0 \cdot 16$ & - & - & 0.94 & 2 & - & 2 \\
\hline $0 \cdot 76-1 \cdot 15$ & 0.58 & 一 & - & $2 \cdot 82$ & 2 & 1 & 1 \\
\hline $1 \cdot 16-1 \cdot 61$ & 0.80 & 2 & - & $4 \cdot 7$ & 6 & 2 & 4 \\
\hline $1 \cdot 62-2 \cdot 34$ & $1 \cdot 6$ & 1 & 2 & $9 \cdot 4$ & 12 & 6 & 6 \\
\hline $2 \cdot 35-3.00$ & $1 \cdot 6$ & - & 1 & $9 \cdot 4$ & 8 & 5 & 3 \\
\hline $3 \cdot 01-4 \cdot 35$ & $3 \cdot 2$ & 6 & 3 & $18 \cdot 8$ & 20 & 10 & 10 \\
\hline $4 \cdot 36-6.06$ & $3 \cdot \overline{2}$ & 3 & 2 & $18 \cdot 8$ & 22 & 13 & 9 \\
\hline $6 \cdot 07-7 \cdot 29$ & $1 \cdot 6$ & 1 & 2 & $9 \cdot 4$ & 5 & 4 & 1 \\
\hline $7 \cdot 30-9 \cdot 24$ & $1 \cdot 6$ & 1 & 2 & $9 \cdot 4$ & 7 & 4 & 3 \\
\hline $9 \cdot 25-11 \cdot 01$ & 0.80 & i & 2 & $4 \cdot 7$ & 6 & 5 & 1 \\
\hline $11 \cdot 02-13 \cdot 39$ & 0.58 & $\mathbf{l}$ & - & $2 \cdot 82$ & 2 & 2 & - \\
\hline $13 \cdot 40-15.09$ & $0 \cdot 16$ & - & 1 & 0.94 & 1 & - & 1 \\
\hline \multirow[t]{2}{*}{$15 \cdot 10_{-}$} & $0 \cdot 16$ & - & 1 & 0.94 & - & - & 一 \\
\hline & $16 \cdot 00$ & 16 & 16 & $94 \cdot 00$ & 94 & 52 & 42 \\
\hline
\end{tabular}

tested. The conformity of the sixteen counts with the Poisson distribution is illustrated in Table I, cols. 2-4. The distribution of $\chi^{2}$ from the N.A.P. and Fildes' counts is compared with the theoretically expected distribution (Fisher, sect. 24). The number of counts is too small to test the fit very well, but the predominance of unduly high values of $\chi^{2}$ from the N.A.P. results is suggestive. In a number of parallel counts that are not included in this analysis, one or more of the six N.A.P. yielded a widely discrepant count, 10-, 100- or a 1000-fold less than the mean of the remaining plates; the plates were all made from the same batch of medium in each case, and no obvious cause, such as the thickness or the $p \mathrm{H}$ of the agar, could be found for the discrepancy. The Fildes' plate results, on the other hand, were remarkably constant throughout.

A second possible criterion of the value of a medium is the size attained by discrete colonies after standard incubation. The most luxuriant growth, however, is not necessarily obtained on media with the greatest capacity to initiate growth. For example, a series of tests, made in another connexion, comparing counts of Brucella melitensis on nutrient and on liver agar, showed that some batches of liver medium failed to support the growth of an 
inoculum that yielded 100 colonies on nutrient agar; yet the growth on liver from 500 nutrientagar-viable organisms was four times as luxuriant as that of the same inoculum on the nutrient agar. Nevertheless, well-developed colonies are useful indications of the goodness of a medium, if judged in conjunction with the count values.

A third criterion is available in the variation in colony size on different media. In general, the worse the medium, the greater the variation. The wide variation induced by the addition to a medium of a partially effective concentration of a bacteriostatic substance is obvious without measurement (e.g. the growth of Brucella abortus on weak thionin media). In less obvious instances, differences in variability are revealed by measurement. For example, we have found that the magnitudes of the coefficient of variation of Meningococcus colony sizes on serum and on haemoglobin agar, in air and in air $+10 \% \mathrm{CO}_{2}$, increased in the same order as the mean colony size and mean count decreased; i.e. $\mathrm{Hb}$ agar in $\mathrm{CO}_{2}>$ serum agar in $\mathrm{CO}_{2}>$ the $\mathrm{Hb}$ agar in air > serum agar in air. Moreover, in these tests and in a series of tests of Haemophilus parainfluenzae on blood and Fildes' medium, the diameters of the largest colonies on the medium with the greater scatter seldom exceeded the mean diameter of those on the small-scatter medium; i.e. the scatter was in the direction of small colonies, indicating a defect of the one medium that resulted in a growth-lag of the less vigorous organisms.

The colony sizes on the six-plate counts were measured under low-power magnification in drop areas containing two to five colonies, and the mean, $\bar{x}$ and s.e. were calculated from fifteen to thirty measurements. The coefficient of variation, c.v., expressed as a percentage, was chosen as the index of scatter: c.v. $=($ S.E. $\times 100) / \bar{x}$.

Table II. Mean colony counts and mean colony sizes of Staph. aureus on 16 batches of nutrient and Fildes' agar

\begin{tabular}{|c|c|c|c|c|c|c|c|}
\hline \multicolumn{3}{|c|}{ Count } & \multicolumn{5}{|c|}{ Colony size (mm.) } \\
\hline $\begin{array}{c}\text { N.A.P. } \\
m_{1}\end{array}$ & $\begin{array}{c}\text { F.A.P. } \\
m_{2}\end{array}$ & $m_{2} / m_{1}$ & $\underset{\bar{x}_{1}}{\text { N.A.P. }}$ & c.v. & $\underset{\bar{x}_{2}}{\text { F.A.P. }}$ & c.v. & $\bar{x}_{2} / \bar{x}_{1}$ \\
\hline $2 \cdot 6$ & $3 \cdot 6$ & 1.49 & 一 & - & 一 & - & - \\
\hline $4 \cdot 2$ & $7 \cdot 3$ & 1.74 & - & - & - & - & - \\
\hline $4 \cdot 7$ & $6 \cdot 3$ & $\mathbf{1} \cdot \mathbf{3 4}$ & - & - & - & - & - \\
\hline $5 \cdot 7$ & $5 \cdot 2$ & 0.90 & - & - & - & - & - \\
\hline $7 \cdot 7$ & $8 \cdot 3$ & 1.08 & 1.04 & $20 \cdot 0$ & 1.06 & $9 \cdot 3$ & 1.02 \\
\hline 11.6 & $15 \cdot 0$ & $1 \cdot 35$ & 0.69 & $10 \cdot 1$ & 0.78 & $10 \cdot 7$ & $1 \cdot 13$ \\
\hline $13 \cdot 3$ & $17 \cdot 0$ & $1 \cdot 28$ & $0 \cdot 56$ & $28 \cdot 2$ & 0.95 & $10 \cdot 3$ & $1 \cdot 70$ \\
\hline $13 \cdot 3$ & $12 \cdot 8$ & 0.96 & $1 \cdot 43$ & $10 \cdot 0$ & $1 \cdot 45$ & $6 \cdot 2$ & $1 \cdot 01$ \\
\hline $14 \cdot 1$ & $16 \cdot 8$ & $1 \cdot 19$ & $1 \cdot 34$ & $13 \cdot 3$ & $1 \cdot 45$ & $6 \cdot 2$ & 1.08 \\
\hline $14 \cdot 3$ & $17 \cdot 1$ & $1 \cdot 20$ & $1 \cdot 69$ & $8 \cdot 1$ & 1.69 & $11 \cdot 1$ & 0.00 \\
\hline 25.7 & 79.2 & $3 \cdot 16$ & 1.67 & $6 \cdot 0$ & $1 \cdot 72$ & $5 \cdot 7$ & $1 \cdot 17$ \\
\hline $34 \cdot 5$ & $37 \cdot 6$ & $\begin{array}{l}1.09 \\
\end{array}$ & 1.08 & $9 \cdot 3$ & $1 \cdot 15$ & $4 \cdot 0$ & $1 \cdot 06$ \\
\hline $39 \cdot 0$ & $46 \cdot 5$ & $1 \cdot 18$ & 1.63 & 6.7 & $1 \cdot 71$ & 3.9 & 1.05 \\
\hline $47 \cdot 2$ & $73 \cdot 0$ & 1.55 & 2.01 & $5 \cdot 0$ & 1.90 & 5.9 & 0.95 \\
\hline 51.5 & 60.0 & $1 \cdot 18$ & 0.94 & $12 \cdot 3$ & 1.04 & $9 \cdot 4$ & $1 \cdot 13$ \\
\hline $53 \cdot 3$ & $60 \cdot 2$ & $1 \cdot 14$ & - & - & - & - & - \\
\hline
\end{tabular}

Of the sixteen batches of media (see Table II), fourteen gave a slightly higher Fildes' count. In the eleven tests of colony size the Fildes' colonies were larger in nine, the same size in one, and less in one. The differences between the corresponding pairs of means, either of counts or colony size, were not individually significant. (The significance of the differences was assessed by the $t$ test, where $t=$ difference/ standard error of difference.) But by calculating the F.A.P. : N.A.P. ratios, both of counts and sizes and treating these as Fisher (sect. 24), the increase in count and mean diameter with the addition of Fildes' extract was such that it would occur by chance once in twenty and 
ten trials respectively, on the assumption that the media did not differ in their effect on Staph. aureus. The average coefficient of variation of colony size was $12.9 \%$ on N.A.P. and only $8.2 \%$ on Fildes; in three of the eleven tests the c.v. was greater on the Fildes, though only by $0 \cdot 6,0 \cdot 9$ and $3.0 \%$. By all the criteria, Fildes' agar was indicated as the medium for Staph. aureus. Fildes' extract, which can be prepared in bulk and kept for some time, apparently acts as a standardizing substance, bringing the more variable heart extract up to a definite level of excellence.

The choice was justified by the analysis of subsequent Fildes' counts. Table I, col. 6, shows the distribution of $\chi^{2}$ calculated from ninety-four sixplate Fildes' counts of Staph. aureus. The fit with the expected distribution in col. 5 is good, for the total $\chi^{2}$ from all counts differs from its expected value, $\sqrt{ }\left(2 \chi^{2}\right)-\sqrt{ }(2 n-1)=0$, by -0.4733 (there are $94(N-1)=470$ degrees of freedom), a deviation to be expected 32 times in 100 such trials. The means of the counts fell into two groups, one containing low values between 2 and 20 , the other high values between 35 and 125. A separation of the values of $\chi^{2}$ from these groups yielded the distributions in cols. 7 and 8 . There is a greater spread of values from the higher means, an effect ascribable to overcrowding. It is only slight, because the $m>35$ distribution shows no marked deviation from the expected distribution. Nevertheless, in practice, drop-area counts of 80 or over were avoided in favour of the counts of 8 or more which arose from the drop areas seeded with the next lower tenfold dilution.

The detailed analysis of the data presented above not only gives several independent indications of the comparative value of a medium, but it provides a sound basis for simplification of the calculations involved in dealing with sampling errors. For if it can be established that the technique yields counts conforming with samples from a Poisson series, the calculation of the s.E. from the individual values of a count may be dispensed with, and assumed to be equal to the square root of the mean count. We shall deal with this point in connexion with the s.E. of the bactericidal power.

\section{The MEASUREMENT OF BACTERICIDAL POWER}

\section{Technique of the bactericidal tests}

Defibrinated blood was distributed in 0.4 c.c. lots in six soft-glass tubes $70 \times 5 \mathrm{~mm}$. internal diameter. The blood was tested within half an hour of its withdrawal from a vein. Each tube received five 0.02 c.c. drops of each of six culture dilutions, and at the same time samples were taken from the dilution tubes for the surface-viable count, thus ensuring a count of the inoculum at the time of mixing with the blood. The resulting 0.5 c.c. fluid column occupied about $20 \mathrm{~mm}$., leaving ample room for the motions of the fluid during mixing. The tubes were sealed with glass plugs cemented in with hard wax ( $R$. Hare, personal communication), mounted in a Todd's (1927 $a$ ) mixing machine, and incubated at $37^{\circ} \mathrm{C}$. After incubation, the contents of the tubes were sampled with standardized dropping pipettes, and six 0.02 c.c. drops of each mixture let fall on to numbered sectors of six well-dried Fildes' agar plates, as in the surface-viable count. Absorption of the blood fluid took place, leaving on the agar surface an opaque red gel in the form of a thin plaque about $15 \mathrm{~mm}$. in diameter (referred to 
below as the "plaque"). The development of staphylococcal colonies in the depths of this gel was slow, and the final size attained by the colony small, but at its edge or on its surface the growth was as rapid as that on a plain agar medium.

Counts of the same plaque made after 18 and $42 \mathrm{hr}$. incubation were not significantly different individually, though $42 \mathrm{hr}$. counts were always higher. In fourteen comparisons of six-plate means, the ratio of 18 to $42 \mathrm{hr}$. counts ranged from $1: 1.02$ to $1: 1 \cdot 5$, with a mean of $1: 1 \cdot 15$, and the difference $(1 \cdot 0-1 \cdot 15=-0 \cdot 15)$ was $2 \cdot 75$ its own standard error. From the $t$ test this difference would arise by chance only once in 70 times, so that the $42 \mathrm{hr}$. counts, as a whole, were significantly higher. $42 \mathrm{hr}$. counts were used in all the following determinations.

Table III. The distribution of $\chi^{2}$ from plaque counts of Staphylococcus blood mixtures, on Fildes' agar

\begin{tabular}{|c|c|c|c|c|c|c|}
\hline$x^{2}$ & Expected & Observed & Expected & Observed & Expected & Observed \\
\hline $0.00-0.55$ & $1 \cdot 79$ & 2 & $0 \cdot 79$ & 1 & 1 & 1 \\
\hline $0.56-0.75$ & $1 \cdot 79$ & 4 & 0.79 & 2 & 1 & 2 \\
\hline $0 \cdot 76-1 \cdot 15$ & $5 \cdot 37$ & 20 & $2 \cdot 37$ & 8 & 3 & 12 \\
\hline $1 \cdot 16-1 \cdot 61$ & 8.95 & 11 & $3 \cdot 95$ & 3 & 5 & 8 \\
\hline $1 \cdot 62-2 \cdot 34$ & $17 \cdot 9$ & 26 & $7 \cdot 9$ & 13 & 10 & 13 \\
\hline $2 \cdot 35-3 \cdot 00$ & $17 \cdot 9$ & 21 & $\mathbf{7 \cdot 9}$ & 5 & 10 & 16 \\
\hline $3 \cdot 01-4 \cdot 35$ & $35 \cdot 8$ & 40 & $15 \cdot 8$ & 21 & 20 & 19 \\
\hline $4 \cdot 36-6 \cdot 06$ & $35 \cdot 8$ & 22 & $15 \cdot 8$ & 9 & 20 & 13 \\
\hline $6 \cdot 07-7 \cdot 29$ & $17 \cdot 9$ & 17 & 7.9 & 11 & 10 & 6 \\
\hline $7 \cdot 30-9 \cdot 24$ & $17 \cdot 9$ & 11 & $\mathbf{7 \cdot 9}$ & 5 & 10 & 6 \\
\hline $9 \cdot 25-11 \cdot 01$ & 8.95 & 5 & 3.95 & 1 & 5 & 4 \\
\hline $11 \cdot 02-13 \cdot 39$ & $5 \cdot 37$ & 0 & $2 \cdot 37$ & 0 & 3 & 0 \\
\hline $13 \cdot 40-15 \cdot 09$ & $1 \cdot 79$ & 0 & $0 \cdot 79$ & 0 & 1 & 0 \\
\hline \multirow[t]{2}{*}{$15 \cdot 10$} & $1 \cdot 79$ & 0 & 0.79 & 0 & 1 & 0 \\
\hline & $179 \cdot 00$ & 179 & $79 \cdot 00$ & 79 & 100 & 100 \\
\hline
\end{tabular}

The plaque counts from 179 six-plate determinations were tested for their goodness of fit to a Poisson series. Table III, cols. 2 and 3, shows the distribution of $\chi^{2}$. There is a marked excess of low values over the high values. The total $\chi^{2}$ from an unselected series of 144 of these counts was 534.10, and $\sqrt{ }\left(2 \chi^{2}\right)-\sqrt{ }(2 n-1)$ is $-5 \cdot 25$ (there are $144(N-1)=720$ degrees of freedom), indicating a significant deviation from the Poisson series. A general antibacterial property of the plaque, such as the persistence of the bactericidal power of the blood after its removal from the tube, would suppress organisms whose vigour lies below a certain level, and the remaining organisms destined to produce visible colonies would still give Poisson counts. The cause of the deviation must therefore be sought for in a variability of growth-promoting properties in different parts of the plaque, such that there is a selective action on certain samples or parts of samples. If, for example, some of the organisms in high-value samples fail to develop, whereas all the organisms develop in lowvalue samples from the same mixture, the result would be a reduction of the standard error of the count from all the samples and a drop in the value of $\chi^{2}$ obtained from it. The selective action is not associated with overcrowding of 
colonies in the plaque, at any rate in the range studied, for the $\chi^{2}$ values are not materially lessened with increasing numbers of colonies in the plaques. The 179 $\chi^{2}$ values in Table III have been divided into two distributions (cols. 4-7), i.e. those from mean plaque counts up to $5 \cdot 0$, and those from counts $5 \cdot 1$ to 75 , and both show similar degrees of asymmetry. The same result was indicated by the series of 144, which, divided into 72 high- and 72 low-value counts, gave $\chi^{2}$ totals of 261.58 and 272.52 respectively, differing by the insignificant amount of 10.94 for 360 degrees of freedom.

There are two practical consequences of this result, whatever its cause may be. In the first place, the low $\chi^{2}$ values indicate that the mean number of organisms surviving the exposure to blood may in fact be greater than the number of colonies that develop in the plaques. In the second place, the square root of the mean will not be as reliable an estimate of the standard error of the plaque counts as it was of the drop-area counts of culture dilution.

\section{The estimation of the bactericidal power}

Bactericidal power is most conveniently indicated by $p$, the proportion of $n$ bacteria that survive mixture with the blood tested. $p=r / n$, where $r$ is the number of bacteria surviving, and the bactericidal power is inversely proportional to the survival rate $p$.

The estimates of $r$ and $n$ are derived from means of surface-viable and plaque counts, both of which are subject to sampling errors. The standard error of $p$ is

$$
\sigma_{p}=\sqrt{ }\left(p q / n N+p^{2} \sigma_{n}{ }^{2} / n^{2}\right)
$$

(see Dr Irwin's note), where $q=(1-p), \sigma_{n}$ is the standard error of $n$ and $N$ the number of plates used in the determination of $r$ and $n$.

The $\chi^{2}$ data justify the use of $\sqrt{ } m$ as a reliable estimate of the standard error of the surface-viable counts, and of $\sqrt{ } m / N$ (where $N=$ the number of plates) as an estimate of the s.E. of the mean count. If $m=n, \sigma_{n}{ }^{2}=n / 6$ and $\sigma_{p}=\sqrt{ }\left(p q / 6 n+p^{2} / 6 n\right)$.

The standard error, $\sigma_{r}$, of the mean of the plaque-counts does not appear in equation (i), and as far as the actual calculation of $\sigma_{p}$ is concerned, need not be known. But $p$ is derived from $r$, and the standard error of $r$ will in part determine the reliability of $p$ as an estimate of the number of bacteria surviving. The $\chi^{2}$ data showed that

(1) the standard error of the plaque counts was usually less than $\sqrt{ } r$, the standard error to be expected if $r$ was derived from a Poisson series, and that

(2) the mean of the plaque count was probably an under-estimate of $r$, the number of bacteria surviving.

As far as (1) is concerned, by adopting $\sqrt{ } r / N(=\sqrt{ } r / 6)$ as the value for $r$, we should, in the majority of cases, be using a larger standard error than that derived from the actual plaque counts, and consequently be increasing the value of a standard error, such as that of $p$, derived indirectly or directly from that quantity. As a result of (2), $p$ will be over-estimated. The over-estimation will, however, be consistent, for the skewness of the $\chi^{2}$ distribution was independent of the magnitude of the mean plaque-counts occurring in the tests. Our object is a comparison of bactericidal powers, and a consistent degree of under-estimation of $r$ will 
not affect the comparison. We have therefore made the assumption that the mean plaque count is a reliable estimate of $r$, and that $r$ has a standard error of $\sqrt{ } r / 6$, though this second value is not used explicitly in our calculations.

Table IV. Relations of formulae for the calculation of $\mathrm{p}$ and $\sigma_{p}$ from the mean counts $\mathrm{m}$ in different dilutions of test culture, and $\mathrm{r}$, the mean plaque count. " $\mathrm{U}$ " indicates an uncountable number of colonies in the drop areas

\begin{tabular}{|c|c|c|c|}
\hline Culture dilution used (say) & $10^{-4}$ & $10^{-5}$ & $10^{-6}$ \\
\hline $\begin{array}{l}\text { Mean of } N(=6) \text { colony counts in } \\
0.02 \text { c.c. culture-dilution }\end{array}$ & “U” & "U" & $m$ \\
\hline $\begin{array}{l}\text { Estimated number of organisms } \\
\text { in } 0.1 \text { c.c. of dilution }\end{array}$ & $500 \mathrm{~m}$ & $50 m$ & $5 m$ \\
\hline $\begin{array}{l}\text { Inoculum }(n) \text { in } 0 \cdot 02 \text { c.c. blood- } \\
\text { organism mixture }(n=k m)\end{array}$ & $20 m$ & $2 m$ & $m / 5$ \\
\hline S.E. of inoculum $\left(\sigma_{n}\right)$ & $20 \sqrt{ } m / N$ & $2 \sqrt{ } m / N$ & $\sqrt{ } m / 25 N$ \\
\hline$\therefore \sigma_{n}=$ & $\sqrt{ } 20 n / 6$ & $\sqrt{ } 2 n / 6$ & $\sqrt{ } n / 30$ \\
\hline Survival rate $(p)$ & $r / 20 m$ & $r / 2 m$ & $\mathbf{5} r / m$ \\
\hline S.E. survival rate $\left(\sigma_{p}\right)$ & $\sqrt{ }\left[\left(p+19 p^{2}\right) / 6 n\right]$ & $\sqrt{ }\left[\left(p+p^{2}\right) / 6 n\right]$ & $\sqrt{ }\left[\left(p-0.8 p^{2}\right) / 6 n\right]$ \\
\hline
\end{tabular}

In the experimental conditions, the mean of the surface-viable count, $m$, is not in fact the value of $n$, the blood inoculum in the test proper. For convenience of description, let the countable number of colonies, $m$, arise from 0.02 c.c. of a $10^{-6}$ dilution (see Table IV); $0 \cdot 1$ c.c. is added to 0.4 c.c. blood, and each blood-drop of 0.02 c.c. contains $m / 5$ organisms. Since bloods of low bactericidal power kill at least $50 \%$ of the cocci, very few organisms develop from such a mixture, and the mean plaque count is unreliable. The $10^{-5}$ dilution of the staphylococcal culture is uncountable (Table IV, " $U$ ") and the drop content is therefore estimated from the $10^{-6}$ count as $10 \mathrm{~m}$; blood drops from mixtures with this dilution contain $2 m$ cocci. Similarly, blood drops made with the $10^{-4}$ dilution contain $20 m$ cocci. Thus $n$ may have the values $m / 5,2 m$, $20 m$, etc., with standard errors $\frac{1}{5} \sqrt{ } m / 6,2 \sqrt{ } m / 6,20 \sqrt{ } m / 6$, etc. The standard error of $n, \sigma_{n}$, will be $\sqrt{ } n / 30, \sqrt{ } 2 n / 6, \sqrt{ } 20 n / 6$, etc., according to the dilution of culture used for the estimation of $m$. Substituting these values of $n$ in equation (i) we have

$$
\text { for } \begin{aligned}
n & =0 \cdot 2 m, & & \sigma_{p}=\sqrt{ }\left[\left(p-0 \cdot 8 p^{2}\right) / n N\right], \\
n & =2 m, & & \sigma_{p}=\sqrt{ }\left[\left(p+p^{2}\right) / n N\right], \\
n & =20 m, & & \sigma_{p}=\sqrt{ }\left[\left(p+19 p^{2}\right) / n N\right] .
\end{aligned}
$$

The relations of these various values are shown in Table IV.

Given $p$ and $\sigma_{p}$, the significance of a difference, $d=\left(p_{1}-p_{2}\right)$, between any two values $p_{1}$ and $p_{2}$ of the survival rate in two bloods, can be estimated in the usual way (Fisher, sect. 24.1). The standard error of the difference, $\sigma_{d}$, is $\sqrt{ }\left({\sigma_{p_{1}}}^{2}+{\sigma_{p_{2}}}^{2}\right)$, and if $d / \sigma_{d}$ is less than 3 , the difference cannot be assumed to have arisen from any significant difference in the bactericidal powers of the two bloods.

As examples of the calculations, the figures in Table VIII 4 , tests 28 and 33, were obtained as follows. In test $28, m$ was $40 \cdot 7$ in the $10^{-5}$ culture dilution. The plaque count, $r$, from the mixture with the $10^{-3}$ culture-dilution, was $18 \cdot 8$. $n$ is therefore $20 m=814$, and $p=18 \cdot 8 / 814$ 
$=0.0231 ; \sigma_{p}^{2}$ is $\left[0.0231+19(0.0231)^{2}\right] / 6 \times 814=0.000006805$ (equation (iv)). In test 33, $m=69.3$ in the $10^{-5}$ dilution; $r=8.2$ in the mixture with the $10^{-4}$ culture-dilution; therefore $n=2 m=138 \cdot 6$, and $p=0 \cdot 0591 ; \sigma_{p}^{2}=\left[0 \cdot 0591+(0 \cdot 0591)^{2}\right] / 6 \times 138 \cdot 6=0 \cdot 00007526$ (equation(iii)). The difference between the two values of $p$ is 0.0360 , and $\sigma_{d}=\sqrt{ }(0.000006805+0.00007526)$ $= \pm 0 \cdot 00906$. The difference, in terms of its own standard error is $d / \sigma_{d}=0 \cdot 0360 / 0 \cdot 00906=3 \cdot 97$, and is therefore significant in the sense defined above.

\section{StANDARDIZATION OF THE TEST}

Rotation period. Five parallel sets of bacterial test-tubes of the same specimen of blood were rotated with the same culture dilutions at $37^{\circ} \mathrm{C}$. for $1,2,4,6$ and $24 \mathrm{hr}$. respectively. Table $\mathrm{V}$ summarizes the result of three experiments with normal bloods of different bactericidal powers. Maximum killing takes place at $2 \mathrm{hr}$; ; staphylococci multiply slowly up to $6 \mathrm{hr}$. and are growing freely at $24 \mathrm{hr}$.

Table V. Effect of time of rotation on $p$; three normal human bloods. Strain $P 900$

\begin{tabular}{cccc} 
Time & \multicolumn{3}{c}{ Experiment } \\
hr. & $\overbrace{1}$ & 2 & 3 \\
1 & $0 \cdot 1610$ & 0.546 & $0 \cdot 1790$ \\
2 & $0 \cdot 0675$ & 0.448 & 0.0413 \\
4 & 0.0733 & 0.675 & 0.0595 \\
6 & 0.0745 & $(1 \cdot 850)$ & 0.0762 \\
24 & Overgrown & Overgrown & Overgrown
\end{tabular}

Strains used. Two strains of Staph. aureus were used, P 802 and P 900. They were recently isolated from acute abscesses, fermented mannitol, liquefied gelatin rapidly and were coagulase and Voges-Proskauer positive. Subcultures of P802, used in the technical tests, were grown each time from $0.75 \%$ nutrient agar stab cultures that had been kept at room temperature and subcultured every 6 weeks. Strain P900 was standardized as follows for the bactericidal tests of human blood. The centrifuged deposit of a young broth culture was distributed into a large number of tubes containing sterile filter paper. The papers were dried in vacuo over $\mathrm{H}_{2} \mathrm{SO}_{4}$ and the tubes sealed with wax and stored in the dark at room temperature. When required, the dry culture was incubated for $18 \mathrm{hr}$. with 5 c.c. broth, and a subculture from this used for the tests.

Age of culture. Parallel tests were made to determine the susceptibility to blood of 3 and $18 \mathrm{hr}$. cultures, both washed and unwashed. Washing consisted of centrifuging the culture and resuspending the packed and drained sediment in fresh warm broth. Tables $\mathrm{VI}_{\mathbf{A}}$ and $\mathbf{B}$ summarize the results of these tests. Three-hour cultures are killed less easily than $18 \mathrm{hr}$. cultures. The work of Lyons (1937) suggests that in $18 \mathrm{hr}$. cultures of Staph. aureus, the cocci are more degraded than in $3 \mathrm{hr}$. cultures. The use of $3 \mathrm{hr}$. cultures is thus indicated in routine bactericidal tests. But since the differences between each of the two values of $p$ in Exps. 4, 5 and 6 is not significant, the more convenient $18 \mathrm{hr}$. culture was used in the majority of the tests. The effect of washing either the 3 or the $18 \mathrm{hr}$. cultures is variable (Exps. 7-12, Table VIв).

Table VIA. Effect of the age of the culture of $\mathrm{p}$; normal human blood. Strain P802

$3 \mathrm{hr}$. culture $18 \mathrm{hr}$. culture

\begin{tabular}{|c|c|c|c|c|c|}
\hline & ir. cult & hr. cult & & & \\
\hline Exp. & $p_{1}$ & $p_{2}$ & $\left(p_{1}-p_{2}\right)$ & $\sigma_{d}$ & $d / \sigma_{d}$ \\
\hline 4 & 0.212 & $0 \cdot 156$ & +0.046 & 0.0247 & $1 \cdot 86$ \\
\hline 5 & $0 \cdot 238$ & $0 \cdot 197$ & +0.041 & 0.0288 & 2 \\
\hline 6 & $0 \cdot 180$ & $0 \cdot 131$ & +0.049 & 0.0226 & 17 \\
\hline
\end{tabular}


Table VIв. Effect of washing the culture on the value of $\mathrm{p}$; normal human blood. Strain P802

$\begin{array}{rcc} & \text { Age } & \\ \text { culture } & \text { Unwashed } \\ \text { Exp. } & \text { hr. } & p_{1} \\ 7 & 18 & 0 \cdot 139 \\ 8 & 18 & 0 \cdot 144 \\ 9 & 18 & 0 \cdot 422 \\ 10 & 3 & 0 \cdot 182 \\ 11 & 3 & 0 \cdot 104 \\ 12 & 3 & 0 \cdot 080\end{array}$

Washed
$p_{2}$
$0 \cdot 163$
$0 \cdot 186$
$0 \cdot 334$
$0 \cdot 130$
$0 \cdot 110$
$0 \cdot 056$

$d$
-0.024
-0.042
+0.088
+0.052
-0.006
+0.024

$\sigma_{d}$
0.0177
0.0208
0.0310
0.0191
0.0208
0.0143

$d / \sigma_{d}$

1.35

$2 \cdot 02$

$2 \cdot 83$

$2 \cdot 72$

$0 \cdot 29$

$1 \cdot 68$

In all the following experiments, the blood was mixed with unwashed $18 \mathrm{hr}$. cultures, rotated at $37^{\circ} \mathrm{C}$. for $2 \mathrm{hr}$, and the plaque colonies counted after $42 \mathrm{hr}$. incubation.

It might be objected that the plaque count measures not only the bactericidal effect of the blood up to the moment of sampling, but also that of the concentrated semisolid blood of the surface of the agar plate. Todd $(1927 b)$, using streptococci, virtually stopped the bactericidal effect at the moment of sampling by diluting the blood-bacterium mixture in molten agar. For an estimation of the rate of killing such a stoppage is essential, either by dilution or by the addition of a neutralizing substance. Experiments with sodium poly-anetholsulphonate (Liquoid) show that it can be used in this way to neutralize a high proportion of the total bactericidal powers of rabbits' blood; and culture experiments in human blood (von Haebler \& Miles, 1938) indicate similar degrees of neutralization. In the recorded experiments the samples were taken at the time of maximum bactericidal effect, after which further incubation in the rotation tubes resulted in multiplication of the cocci. At this stage little bactericidal power remains, for in a small series of tests the plaque count was not significantly altered by the addition of $0.05 \%$ of Liquoid to the blood-bacterium mixture immediately before sampling for the plaque count.

\section{Fallacies in the interpretation of $p$}

The number of colonies developing in the blood plaques may be decreased as a result of aggregation of the cocci by at least two mechanisms, phagocytosis and agglutination.

Aggregation by phagocytosis. Two or more organisms, each destined to survive as a colony producer whether ingested by a leucocyte or not, may be gathered into one leucocyte and give rise to one colony only.

Some idea of the magnitude of this error may be gained from the following considerations. We may assume highly favourable conditions for phagocytosis, i.e. that the blood contains as many as 20,000 equally phagocytic w.B.c. per c.mm., that contact with a coccus results in its ingestion by the w.B.c., and that all the cocci are so ingested. The number of such w.B.c. in 0.02 c.c. will be 400,000 . An inoculum of 400 cocci in this volume represents an average bactericidal mixture. Initially there are 1000 w.B.c. to each coccus, and since phagocytosis cannot take place in the free fluid except by chance approximation of w.B.c. and coccus, the probabilities, $P$, of any given w.B.c. ingesting 
0,1 or 2 bacteria will be the first three terms of a Poisson series, in which $M=1 / 1000$, the ratio of bacteria to w.B.c. These are:

$\begin{array}{cccc}\text { No. ingested } & 0 & 1 & 2 \\ P & 0.99900 & 0.0009990 & 0.000000119950\end{array}$

i.e. the odds against one leucocyte ingesting two organisms are over 2 million to 1 . But if an inoculum large enough to equal the number of w.B.c. is used, the probabilities are as follows:

$\begin{array}{ccccc}\text { No. ingested } & 0 & 1 & 2 & 3 \\ P & 0.3679 & 0.3679 & 0.1839 & 0.0613\end{array}$

i.e. $18 \%$ of the cells may contain two, and $6 \%$ may contain three organisms.

Two rotation experiments, mixing $B r$. melitensis with defibrinated rabbit blood and a highly opsonic anti-melitensis serum, illustrated the effect of non-lethal phagocytosis on large and small inocula. There was no reduction in count of the small inocula of 5-40 per 0.02 c.c.; $p$ in fact was 1.0 . But the blood plaques from mixtures incubated with 100 and 1000 times this amount, in which confluent growth might have been expected, also yielded discrete and countable colonies. These colonies were considerably larger than the average. All the w.B.c. in stained smears of the heavily inoculated blood-organism mixtures contained large numbers of bacilli, an effect in part due to the high proportion of bacilli to w.B.c., and from these collections of living bacilli the large colonies presumably developed. The results in the more dilute mixtures showed that the phagocytosis was not lethal to any large degree; for, since the chance of an organism meeting a w.B.c. in unit time is the same in both mixtures there is no reason to suppose that the active phagocytosis deducible from the smears of the concentrated mixture did not operate in the mixtures containing only a few bacteria. The result is comparable with the "epiphylactic" phenomenon of Wright et al. (1923) but differs in that it was observed, not immediately after mixing blood and culture, but $2 \mathrm{hr}$. later. There is no necessity in this case, therefore, for postulating any cause other than the known one of aggregation in leucocytes.

Aggregation by agglutination. The circumstances favourable for this effect would be the presence of sufficient agglutinin to sensitize each bacterium rapidly without producing the "prozone" inhibition of excess antibody, and the absence of lethal or lytic effects dependent on specific antibody sensitization. The velocity of agglutination then depends on the concentration of bacteria.

We have no evidence to offer on staphylococci, but experiments with an organism of similar size, Br. melitensis, have shown that the time $(t)$ of particulation with an optimally particulating concentration of antiserum is inversely proportional to the concentration, $C$, of the bacterial suspension, i.e. $t=K / C$. This relationship held when $C$, measured in terms of organism concentration, was reduced from $2 \times 10^{9}$ per c.c. to $10^{9} / 4$ per c.c. Below values of $10^{9} / 4$ per c.c., $K$ increased with each reduction of $C$. It may be applied, nevertheless, with some confidence to a system like the dilute suspension of cocci in a bactericidal mixture, for the particulation time is likely to be much larger than that given by $t=K / C$ when $K$ is determined for high concentrations of cocci. We shall therefore be over-estimating rather than underestimating the effect of agglutinins in producing aggregation. A $10^{9}$ per c.c. suspension of Staph. aureus mixed with an optimal amount of antiserum is finely particulated into aggre- 
gates of several hundred cocci in about $60 \mathrm{~min}$. at $37^{\circ} \mathrm{C}$. If we allow as little as $0.5 \mathrm{~min}$. of the 60 for sensitization of the cocci and for the formation of aggregates of two cocci in such a mixture, then this degree of aggregation would occur in a concentration of $5 \times 10^{3} \mathrm{staphylococci}$ per c.c. (i.e. that in a strong bactericidal mixture) only after $0.5 \times 10^{9} / 5 \times 10^{3} \mathrm{~min} .=$ approx. $1500 \mathrm{hr}$. This estimate is a rough indication only of the magnitude of specific aggregation velocities, and we have no knowledge of factors which may operate in low concentrations. For example, it is possible that the rate may be increased with living flagellated bacteria, partly because their independent motion will increase chances of contact with each other, and partly because of their greater effective length and surface for sticking together after chance contact.

We have attempted to measure the effect of agglutinins in bacteriumantiserum mixtures, set up as bactericidal tests, compared with control normal serum-bacteria mixtures. Two systems were used-H. parainfluenzae and Bact. coli, with their respective complement-free rabbit antisera. In the tests on the first, the antisera were diluted in saline. In the coli-anticoli systems, the antisera were diluted in $1.0 \%$ heated normal rabbit serum, to obtain a constant concentration of serum proteins. Exps. 13-19 are summarized in Table VII, where the average numbers of bacteria in 0.02 c.c. of each serum-bacterium mixture are recorded. In the parainfluenza experiments, 13-16, the initial

Table VII. Mean colony counts in 0.02 c.c. of culture + homologous antiserum mixtures after $2 \mathrm{hr}$. rotation

\begin{tabular}{|c|c|c|c|c|c|c|c|c|c|}
\hline \multirow[b]{2}{*}{ Exp. } & \multicolumn{6}{|c|}{ H. parainfluenzae } & \multicolumn{3}{|c|}{ Bact. coli } \\
\hline & (13) & (14) & $(15 b)$ & $(15 a)$ & $(16 b)$ & $(16 a)$ & $(17)$ & (18) & $(19)$ \\
\hline Original inoculum & 144 & 20 & 76 & 76 & 61 & 61 & 1 & 1 & 1 \\
\hline Control serum & 34 & 15 & 17 & 31 & 15 & 12 & 140 & 137 & 157 \\
\hline Antiserum: & & & & & & & & & \\
\hline 1 in 24300 & - & 18 & 18 & 16 & 24 & 13 & 273 & 110 & 68 \\
\hline 1 in 8100 & - & 17 & 25 & 33 & 54 & 25 & 83 & 128 & 233 \\
\hline 1 in 2700 & - & 15 & 61 & 65 & 99 & 15 & 400 & 108 & 217 \\
\hline 1 in 900 & 34 & 20 & 121 & 93 & 105 & 45 & 73 & 58 & 207 \\
\hline 1 in 300 & 52 & 22 & 148 & 119 & 560 & 213 & 77 & 68 & 54 \\
\hline 1 in 100 & 89 & 26 & 132 & 119 & 1073 & 800 & 19 & 18 & 10 \\
\hline 1 in 33 & 130 & - & - & - & - & - & 一 & - & - \\
\hline
\end{tabular}

number of viable organisms is reduced in the weak antiserum. The reduction is not due to agglutination, since it occurs in the control serum. With increasing strength of antiserum the count returns to the inoculum value, and exceeds it in the highest concentrations. Exps. 15 and 16 were modified in that after incubation two sets of samples were taken from the rotation tubes, $(a)$ before and $(b)$ after violent agitation with a pipette.

Forcible agitation in 15 increased the strong serum counts, but not significantly. In 16 there was a significant increase, especially marked in counts equal to or greater than the inoculum, which suggests that disintegrable bacterial clumps are formed in the mixtures containing multiplying organisms. The H. parainfluenzae formed granular masses in broth cultures, and the 
clumps may have been incompletely separated daughter cells. On the other hand, daughter cells may have failed to separate into single colony-forming units because they were sensitized by antibody, and so multiplied in agglutinated masses. The Bact. coli experiments, 17-19, support this view. The serum protein content was constant in all mixtures, and the strain of coli grew fully dispersed in fluid cultures, i.e. the daughter cells had no inherent tendency to stick together. In all three experiments the growth was so rapid that countable numbers of colonies were produced only in serum-bacterium mixtures initially seeded with 50 organisms per c.c. In 18, the growth was greatest in the control, and least in concentrated serum. In 17 and 19 there was an initial increase with increasing antibody concentration, followed by a marked decrease. The decrease may have been due to a genuine lethal action of the antiserum; but if agglutination was the cause, the organisms were in all probability agglutinated as they divided, since even in the strongest antibody solution there was 10-20-fold increase of the original inoculum. It is unlikely that the increase represents the growth of a few free organisms not included in an initially agglutinated mass. This might happen if the available agglutinins were completely absorbed by a proportion of the bacteria. But the agglutinin content of the two sera was well in excess of the amount required to sensitize the maximum number of bacilli counted; the titres were $1: 640$ and $1: 5120$ respectively, i.e. concentrations capable of agglutinating $10^{9}$ organisms per c.c.

The results suggest that specific agglutinins can exert an apparently bacteriostatic action on multiplying organisms by keeping the daughter cells in clumps, but there is no evidence of direct clumping of the small numbers of organisms initially dispersed in the antiserum, in the $2 \mathrm{hr}$. rotation period of the bactericidal tests.

It is open to question whether similar results would be obtained if the experiments could be made in blood-bacterium mixtures. The initiation of agglutination and phagocytosis depends on close approximations of one bacterium with another, or with a w.B.c., and the presence of $5 \times 10^{9}$ R.B.c. per c.c. may interfere with such approximations. Two particles in a fluid move towards one another as a result either of Brownian movement or shearing distortions of the particular piece of fluid that contains them. A body of fluid occupying the position of a R.B.c. between two cocci a few microns apart would allow the approximation of the cocci should they be driven together by chance molecular bombardments, but an actual R.B.c. in this position would interfere with it and, by reason of its inertia and size, would tend to remain in the interfering position. On the other hand, R.B.c.'s would interfere very little with approximations due to the shearing movements of a well-agitated fluid; they would behave as particles of fluid of the size and volume of a red cell, round which the actual fluid would slide with ease. It seems not unlikely that the presence of R.B.c.'s reduces the rate of agglutination and phagocytosis in blood-bacterium mixtures by a small and as yet undetermined amount depending on the relative importance of Brownian and mixing movements in securing approximation.

Further evidence on these points is needed. In its absence, it seems reasonable to assume that, in mixtures containing small inocula, fallaciously large degrees of bactericidal power are unlikely to occur as the result of mechanical 
aggregation of colony forming units. Agglutination and non-lethal phagocytosis may reduce the colony counts of large inocula. The bactericidal test described allows an adequate check on such effects. If the reduction of a large inoculum exceeds that of the corresponding inoculum one-tenth or onehundredth its size, i.e. if $p$ decreases with increasing inoculum, then hypotheses, e.g., of mechanical aggregation and of epithylactic responses must be considered. In our experiments, the two values of $p$ calculated from mixtures made with adjacent culture-dilutions of the 10 -fold series never differed significantly. From 40 unselected determinations of normal values of $p$ made from inocula between 5 and 8 , and from the corresponding inoculum of 50-80, the average difference was 0.05 , a value equal only to one-half the standard error of the distribution of normal values.

\section{NORMAL AND ABNORMAL BACTERICIDAL POWER}

In Table VIII A are listed the results of tests on the blood of healthy and of infected adults, with the strains P 802 and P 900. There are five groups of values for $p$ : group I, those of eleven healthy adults tested with P900; group II, those of six healthy adults tested with P 802; group III, those of nine determinations on the blood of one healthy adult, at intervals in a period of ten weeks; group IV, those of seven persons with chronic staphylococcal infections, some of them under treatment with staphylococcal toxoid; and group V, those of two persons with acute Streptococcus pyogenes cellulitis.

In Table VIII в are recorded the differences between various selected pairs of results within the first four groups, expressed in terms of the standard error of the differences (i.e. the ratio $d / \sigma_{d}$ ). If the ratio is in excess of $3 \cdot 0$, the two different values of $p$ concerned cannot be said to have arisen by chance from tests on bloods of essentially the same bactericidal power. The demonstration of significant differences is therefore evidence of the heterogeneity of the bloods tested, as far as their bactericidal power is concerned.

The differences between extreme values, and between extreme values and those lying closest to the average value of $p$ for the group, have been calculated. For example, tests 7 and 8 are nearest to the mean $0 \cdot 143$ of group I, and test $27 a$ to the mean 0.0331 of group IV. In group III all the first eight values differ significantly from the last (test 26). This outstanding value of 0.422 was determined in blood taken after a day's "malaise and insomnia" (recorded before the result of the test was known), and may be excluded as a possibly abnormal value. The extremes (18 and 25 ) of the remainder differ significantly, i.e. the bactericidal power of the blood of one person may undergo significant fluctuations, and until the limits of fluctuations of $p$ for a healthy person are known, the inclusion of a single "normal" blood in a series of bactericidal tests, as a means of fixing "normal" bactericidal power for a given experiment, is an unreliable procedure.

The majority of selected pairs tested in each group yield ratios $>3$, and 
Table VIII A. Values of $p$; the blood of adult human beings

\begin{tabular}{|c|c|c|c|c|}
\hline No. of test & Source of blood & $\begin{array}{l}\text { Group; and strain of } \\
\text { Staphylococcus used }\end{array}$ & $p$ & $\sigma_{p}$ \\
\hline $\left.\begin{array}{r}1 \\
2 \\
3 \\
4 \\
5 \\
6 \\
7 \\
8 \\
9 \\
10 \\
11\end{array}\right\}$ & \multirow[t]{2}{*}{17 healthy adults } & $\begin{array}{l}\text { Group I } \\
\text { (P 900) }\end{array}$ & $\begin{array}{l}0.061 \\
0 \cdot 062 \\
0 \cdot 070 \\
0 \cdot 087 \\
0 \cdot 108 \\
0 \cdot 114 \\
0 \cdot 125 \\
0 \cdot 166 \\
0 \cdot 168 \\
0 \cdot 219 \\
0 \cdot 294\end{array}$ & $\begin{array}{l}0 \cdot 0000879 \\
0 \cdot 0001174 \\
0 \cdot 0001592 \\
0 \cdot 0001687 \\
0 \cdot 0002543 \\
0 \cdot 0001726 \\
0 \cdot 0002130 \\
0 \cdot 0002932 \\
0 \cdot 0002667 \\
0 \cdot 0005675 \\
0 \cdot 0005764\end{array}$ \\
\hline $\begin{array}{l}12 \\
13 \\
14 \\
15 \\
16 \\
17\end{array}$ & & $\begin{array}{l}\text { Group II } \\
\text { (P 802) }\end{array}$ & $\begin{array}{l}0 \cdot 100 \\
0 \cdot 139 \\
0 \cdot 164 \\
0 \cdot 168 \\
0 \cdot 252 \\
0 \cdot 269\end{array}$ & $\begin{array}{l}0.0001198 \\
0 \cdot 0001724 \\
0 \cdot 0001944 \\
0 \cdot 0001999 \\
0 \cdot 0003214 \\
0.0003718\end{array}$ \\
\hline $\left.\begin{array}{l}18 \\
19 \\
20 \\
21 \\
22 \\
23 \\
24 \\
25 \\
26\end{array}\right\}$ & $\begin{array}{l}\text { Nine specimens } \\
\text { from one healthy } \\
\text { adult, during a } \\
\text { l0-week period }\end{array}$ & $\underset{(\text { P 802) }}{\text { Group III }}$ & $\begin{array}{l}0 \cdot 080 \\
0 \cdot 104 \\
0 \cdot 139 \\
0 \cdot 144 \\
0 \cdot 156 \\
0 \cdot 164 \\
0 \cdot 182 \\
0 \cdot 197 \\
0 \cdot 422\end{array}$ & $\begin{array}{l}0 \cdot 0001406 \\
0 \cdot 0002422 \\
0 \cdot 0001510 \\
0 \cdot 0002018 \\
0 \cdot 0001747 \\
0 \cdot 0001944 \\
0 \cdot 0002445 \\
0 \cdot 0003037 \\
0 \cdot 0005392\end{array}$ \\
\hline $\left.\begin{array}{l}27 b \\
27 c \\
28 \\
29 \\
30 \\
31 a \\
27 a \\
32 \\
33 \\
31 b\end{array}\right\}$ & $\begin{array}{l}\text { Patients with } \\
\text { Staph. aureus } \\
\text { infection }\end{array}$ & $\begin{array}{l}\text { Group IV } \\
\text { (P900) }\end{array}$ & $\begin{array}{l}0.0143 \\
0 \cdot 0158 \\
0.0231 \\
0.0262 \\
0 \cdot 0280 \\
0.0305 \\
0.0315 \\
0.0350 \\
0.0591 \\
0.0665\end{array}$ & $\begin{array}{l}0 \cdot 000003929 \\
0 \cdot 000005064 \\
0 \cdot 000006805 \\
0 \cdot 000008175 \\
0 \cdot 000008274 \\
0 \cdot 000009292 \\
0 \cdot 000009457 \\
0 \cdot 000013054 \\
0.00007526 \\
0 \cdot 00003258\end{array}$ \\
\hline $\left.\begin{array}{l}34 \\
35\end{array}\right\}$ & $\begin{array}{l}\text { Patients with Str. } \\
\text { pyogenes cellulitis }\end{array}$ & $\left\{\begin{array}{c}\text { Group } V \\
(\mathrm{P} 900)\end{array}\right.$ & $\begin{array}{l}0 \cdot 434 \\
0 \cdot 357\end{array}$ & $\begin{array}{l}0 \cdot 0007483 \\
0 \cdot 0005825\end{array}$ \\
\hline
\end{tabular}

Table VIII в. The ratio $d / \sigma_{d}$ for differenees between the values of $p$ in selected pairs of tests from Table. VIII A

\begin{tabular}{|c|c|c|}
\hline Group & Tests & $d / \sigma_{d}$ \\
\hline I & $\left\{\begin{array}{l}1 \text { and } 11 \\
1 \text { and } 7 \\
1 \text { and } 8 \\
7 \text { and } 11 \\
8 \text { and } 11\end{array}\right.$ & $\begin{array}{l}9 \cdot 03 \\
3 \cdot 69 \\
5 \cdot 38 \\
6 \cdot 01 \\
4 \cdot 34\end{array}$ \\
\hline II & 12 and 17 & $4 \cdot 33$ \\
\hline III & 18 and 25 & $5 \cdot 57$ \\
\hline IV & $\left\{\begin{array}{l}27 b \text { and } 31 b \\
27 b \text { and } 27 a \\
27 a \text { and } 31 b \\
27 a \text { and } 27 b \\
27 a \text { and } 27 c \\
31 a \text { and } 31 b\end{array}\right.$ & $\begin{array}{l}5 \cdot 40 \\
4 \cdot 70 \\
2 \cdot 88 \\
4 \cdot 70 \\
3 \cdot 88 \\
5 \cdot 41\end{array}$ \\
\hline
\end{tabular}


the values of $p$ represent a series of individual responses to Staph. aureus. The wide variation is not due to day-to-day changes in experimental conditions, for significantly different values were obtained from bloods tested at one time with the same culture dilutions, batch of medium, etc. The number of tests is small, but there are indications that the lower limit of $p$, in healthy adults not recently convalescent from staphylococcal infections, may prove to be in the region of 0.05 for Staph. aureus strains similar to those used in the above tests.

The blood of Staphylococcus-infected patients undergoes changes during the course of the disease. Blood 27 (group IV) from a patient with chronic osteomyelitis, was tested on three occasions: $(a) 5$ months after a first course of toxoid, and immediately prior to a second course, $(b)$ and $(c), 1$ and 4 weeks after the second course had been started. $27 b$ and $c$ are both significantly different from $a$, though not from each other.

$31 a$ and $b$, taken respectively in the active and convalescent stages of a staphylococcal breast abscess, differ by 0.035 , which is 5.41 times its standard error.

The two "streptococcal" values in group V (34 and 35) are well above the mean value for normal bloods.

\section{SUMMARY}

The survival rate, $p$, of a measured inoculum of Staph. aureus in a standard volume of defibrinated blood, is a reliable quantitative measure of the bactericidal power of blood. The number of viable organisms in the inoculum and in the blood-bacterium mixture may be estimated with the necessary accuracy by counts of colonies developing from measured volumes of the fluids let fall on to the surface of solid media. Fildes' agar was the most suitable medium for this surface-viable count, and was selected on the basis of four criteria; of the media tested it yielded the highest counts, and the counts conformed most closely to a Poisson series; and on it the mean colony size was maximum, and the coefficient of variation of colony size was minimum. On this medium, the close conformity of the separate count values to a Poisson series enabled the standard error of the survival rate to be determined from a simplification of the general expression for the standard error of a ratio.

The number of colonies growing from a sample of a blood-bacterium mixture may be reduced, not by killing of the individual cocci, but as a result of their aggregation either by agglutinins in the blood, or in the cytoplasm of leucocytes that are phagocytic but not bactericidal. It appears that these mechanisms are unlikely to operate in blood-bacterium mixture containing relatively few organisms; in such mixtures the survival rate is a reflexion of the killing power only.

The immunological significance of $p$ has not been investigated, but the range of values for healthy human adults differs significantly from that for sufferers from chronic staphylococcal infection. Moreover, by the technique 
employed differences may be detected between individual values of $p$ that cannot reasonably be attributed to technical or sampling errors.

Acknowledgement. We are greatly indebted to Dr J. O. Irwin for his criticisms and for his help with the statistical problems of this investigation.

\section{REFERENCES}

Fisher, R. A. (1930). Statistical Methods for Research Workers. Edinburgh and London. Fisher, R. A., Thornton, H. G. \& Mackenzie, W. A. (1922). Ann. appl. Biol. 9, 35. von Haebler, T. \& Miles, A. A. (1938). J. Path. Bact. 46, 245.

Kenny, M., Johnston, F. D. \& von Haebler, T. (1937). Lancet, $2,119$.

Lyons, C. (1937). Brit. J. exp. Path. 18, 411.

Mackie, T. J. \& Finkelstein (1931). J. Hyg., Camb., 31, 35.

Todd, E. W. (1927a). Brit. J. exp. Path. 8, 1.

- (1927b). Brit. J. exp. Path. 8, 326.

WILson, G. S. (1922). J. Bact. 7, 405.

- (1935). Spec. Rep. Ser. med. Res. Coun., Lond., no. 206.

Wright, A. E., Colebrook, L. \& Storer, E. J. (1923). Lancet, 1, 365, 417 and 473.

Wright, Hedley D. (1933). J. Path. Bact. 37, 257.

\section{A NOTE ON THE STANDARD ERROR OF THE SURVIVAL RATE OF BACTERIA IN BLOOD-BACTERIUM MIXTURES}

\section{By J. O. Irwin}

$n$ bacteria are added to the blood, and $r$ survive. $r$ and $n$ are derived from the mean of $N$ plates, and are subject to error.

The survival rate $p=r / n$.

Taking logarithmic differentials

$$
\frac{\delta p}{p}=\frac{\delta r}{r}-\frac{\delta n}{n}
$$

whence, writing $V(n)$ for the sampling variance of $n$, etc.,

and

$$
\begin{gathered}
\frac{V(p)}{p^{2}}=\frac{V(r)}{r^{2}}+\frac{V(n)}{n^{2}}, \\
V(p)=\frac{p^{2}}{r^{2}} V(r)+\frac{p^{2}}{n^{2}} V(n), \\
V(r)=n p q / N,
\end{gathered}
$$

$$
V(p)=\frac{p q}{n N}+\frac{p^{2}}{n^{2}} V(n)
$$

i.e.

$$
\sigma_{p}^{2}=p q / n N+p^{2} \sigma_{n}^{2} / n^{2} \text {. }
$$


In the tests described above, $n=k m, k$ being a constant depending on the dilution of culture, and $m$ the mean number of colonies counted. If $m$ is the mean of a Poisson series, we have

$$
\begin{aligned}
\sigma_{n}^{2} & =\frac{k^{2} m}{N} \\
& =\frac{n^{2}}{m N} .
\end{aligned}
$$

Hence

$$
\begin{aligned}
\sigma_{p} & =\sqrt{ }\left(\frac{p q}{n N}+\frac{p^{2}}{m N}\right) \\
& =\sqrt{ }\left[\left\{p+(k-1) p^{2}\right\} / n N\right] .
\end{aligned}
$$

(MS. received for publication 6. vIII. 38.-Ed.) 